\title{
BRAIN ABSCESS FOLLOWING ISCHEMIC STROKE WITH SECONDARY HEMORRHAGE
}

\author{
Jorge Luiz Kraemer', Paulo Valdeci Worm², Mário de Barros Faria², Alexandre Maulaz ${ }^{3}$
}

Cerebral vascular events constitute the most common group of neurological diseases, and are usually followed by systemic infections, being pneumonia, urinary tract infection and sepsis the most frequent ${ }^{1}$. Brain abscess compromising the infarcted cerebral tissue is a rare condition ${ }^{2,3}$. It most frequently happens a few weeks after an infectious systemic insult, which had usually followed the ischemic stroke. There are only a few case reports on this subject in the literature. In spite of that, those patients with ischemic stroke should be considered at high risk of developing brain abscess, and should have this condition included in their differential diagnosis once neurologic or even systemic deterioration takes place following previous recovery. Cerebral ischemia is considered to be a predisposing factor for the genesis of brain abscess after stroke ${ }^{4}$. Due to impaired cerebral oxigenation and blood brain barrier disruption in the infarcted area, the formation of an abscess becomes feasible following bacteremia, a fact that has recently caught the attention of neurologists and neurosurgeons $s^{2,3,5}$.

\section{CASE}

A previously healthy 33-year-old white woman is admited to the emergency room complaining of severe right hemiparesis of acute onset and motor disphasia, which progressed to complete aphasia in a few minutes. Brain computed tomography (CT) revealed no abnormalities. Due to the hypothesis of acute obstruction of the left middle cerebral artery (MCA), a cerebral angiography was performed five hours after the onset of the symptoms, indicating oclusion of the distal portion of the left intracranial carotid artery, compromising both anterior and middle cerebral arteries. Intra-arterial thrombolytics were administered, with total reperfusion of the anterior cerebral artery territory. A MCA suboclusion (M2 segment) remained, despite multiple attempts to perform angioplasty with stent deployment. Patient was transferred to the intensive care unit (ICU) and was started on intravenous anticoagulants. About 24 hours after the procedure, patient developed anisocoria with left my- driasis, and tomographic evidence of hemispheric edema compromising the left MCA distribution with intracerebral hemorrhage and significant mass effect. A decompressive craniotomy was performed, and a ventricular catheter for intracranial pressure (ICP) measurement was placed (Fig 1).

Elevated ICP was managed with mannitol and hypertonic saline. Four days after the ictus, patient developed fever $\left(38^{\circ} \mathrm{C}\right)$ with negative blood cultures. Pseudomonas aeruginosa and Staphylococcus aureus were then isolated in the tracheal aspirate, being both ciprofloxacin sensitive. Patient persisted febrile after seven days of antimicrobial therapy, and the previous germs were both once again isolated in the tracheal aspirate. Anaerobic gram positive cocci disposed in chains were identified in the blood culture; piperacilin and tazobactan for ciprofloxacin resistant pseudomonas were introduced, with favorable outcome. Seven weeks after the stroke, patient started with low grade fever and local swelling over the decompressive craniotomy. Brain CT revealed a multiloculated hypodense lesion over the previously infarcted area, with mass effect and peripheral enhancement after contrast injection (Figs 2A and B), suggesting cerebral abscess. Eritrocyte sedimentation rate was elevated and blood count showed microcytosis and hypocromia, with mild leukocytosis and left shift. Transesophagic echocardiography revealed no abnormalities, similar to the one performed on admission. By means of a small skin incision, abscess punture was performed, with drainage of $40 \mathrm{ml}$ of purulent secretion whose culture in anaerobic media revealed gram negative cocci organized in chains, identified as group C Streptococcus sp. Patient was treated with vancomycin and imipenem for 10 weeks, showing good response. She remains hemiparetic, but follows commands and articulates words.

The area of cranial decompression became flacid, and control CT showed the infarcted territory still containing the enhanced abscess capsule. The bone flap, which had been kept transitorially in the subcutaneous fat of the abdomen, was replaced 12 months after the stroke, and the tomographic control demonstrated the left encephalomalatic area with complete resolution of the abscess (Fig 3).

\footnotetext{
ABSCESSO CEREBRAL APÓS INFARTO ISQUÊMICO COM TRANSFORMAÇÃO HEMORRÁGICA

Hospital São José, Santa Casa de Misericórdia de Porto Alegre, Porto Alegre RS, Brazil: 'MD, PhD, Professor of Neurological Surgery, Department of Neurosurgery, Hospital São José - Santa Casa de Misericórdia de Porto Alegre; ${ }^{2}$ MD, Resident in Neurological Surgery, Hospital São José - Santa Casa de Misericórdia de Porto Alegre; ${ }^{3} \mathrm{MD}$, Neurologist, Department of Neurology, Policlínica Santa Clara - Santa Casa de Misericórdia de Porto Alegre.
}

Received 31 August 2007, received in final form 21 November 2007. Accepted 3 December 2007.

Dr. Mário de Barros Faria - Rua Engenheiro Olavo Nunes 153 / 301 - 90440-170 Porto Alegre RS - Brasil. E-mail: barrosfaria@gmail.com 


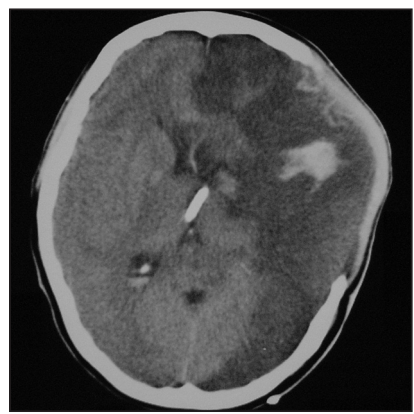

Fig 1. Non-enhanced CT obtained following decompressive craniotomy, demonstrating massive left hemispheric ischemia with central area of hemorrhage and surrounding edema; ventricular catheter for ICP measurement placed in the third ventricle.

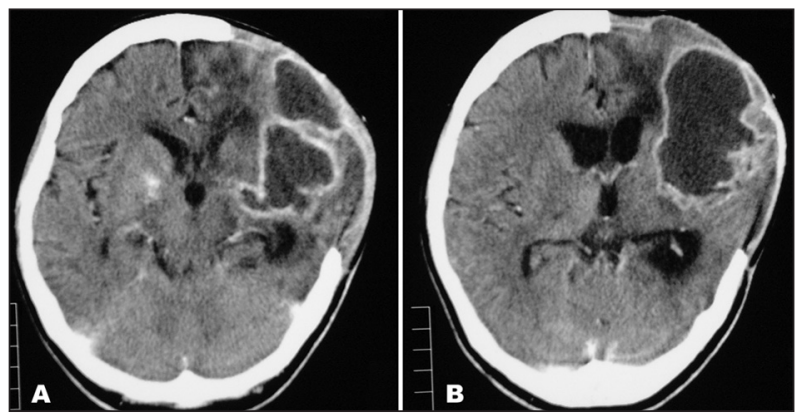

Fig 2. (A) Contrast enhanced CT, revealing an irregular hipodense and multiloculated lesion with sharp peripheral enhancement compromising the infarcted territory. The image is compatible with brain abscess, and protrudes from the cranial defect (B).

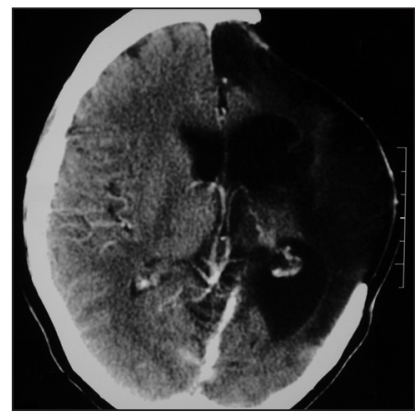

Fig 3. Tomographic control 12 months after the stroke showing left hemispheric area of encephalomalacia, with complete resolution of the abscess.

\section{DISCUSSION}

The acute onset of right hemiparesis, aphasia and impaired consciousness on admission suggested a massive ischemic stroke compromising the cerebral territory supplied by the left MCA. Despite the abscence of tomographic confirmation, performed early in the course of the disease, the characteristic presentation prompted the medical team to attempt reperfusion of the obtructed vessel by means of endovascular techniques. The second CT already demonstrated the infarcted area with significant mass effect and midline shift, which in association with aditional impairement of consciousness, revealed the necessity for a decompressive craniotomy and ICP measurement (Fig 1). The clinical course progressed in a slow pace, complicated by infectious events and long term ICU admission.

There is no pathognomonic syndrome for the presentation of brain abscess, and because of that it is frequently misdiagnosed as neoplasia or other intracranial mass lesion $^{6}$. Headache, fever and impaired consciousness are the most prevalent presenting symptoms, although this triad is found in less than one third of the patients with brain abscess ${ }^{7,8}$. The intensity, or even abscence, of fever is variable among patients, depending on their age, immunologic state and type of etiologic agent involved in the genesis of the abscess. The diagnosis of this entity in patients with massive ischemic or hemorrhagic stroke becomes a challenge to the attending physician due to the similarities between those clinical pictures. The presenting symptoms of both pathologies overlap along the clinical course of those diseases, leading to a clinical scenario in which the development of a brain abscess over a previously infarcted cerebral region produces no additional deficit, ${ }^{9}$ thus delaying diagnosis. A patient with extensive cerebral stroke may be obtunded, aphasic, incapable of complaining of headache. Commonly, he or she has already presented evident motor deficit, and may be febrile due to infectious complications secondary to prolonged hospital admission. The recognition of a brain abscess in a patient like this becomes an exclusion diagnosis, and due to its low frequency, it relies on a high index of suspicion for this specific entity in this group of patients.

The pathogenesis of brain abscess over an infarcted area involves previous local brain damage and a distant focus of infection. The vascular event, ischemic or hemorrhagic, produces local blood brain barrier disruption, which makes this region vulnerable to microbial seeding in the event of bacteremia secondary to systemic infection, leading to the formation of cerebral abscess ${ }^{3}$. Staphylococcus aureus is reported in the literature as the most frequent etiologic agent. In this report, patient had a respiratory tract infection caused by Pseudomonas aeruginosa and Staphylococcus aureus, blood culture positive for anaerobic gram-positive cocci in chains, and Streptococcus $s p$ isolated in the abscess secretion. Brain abscess developed five weeks after the stroke, although it took longer to be diagnosed in more than $70 \%$ of the cases reported in the literature ${ }^{9-15}$. The reduced delivery of antibiotics to ischemic areas decreases the effectiveness of treatment and prolongs its course ${ }^{16,17}$. Kaplan', however, reported three cases with favorable response to eight weeks of antimicrobial therapy. The treatment of brain abscess usually involves surgical drainage, performed whether by stereotactic puncture and aspiration or open surgical resection of the abscess and its capsule, followed by antimicrobial therapy for six to eight weeks. Mampalam and Rosenblum $^{18}$ reported positive cultures in $96 \%$ of the untreated patients, in contrast to $70 \%$ in those who have received 
any antimicrobial therapy before collecting the abscess content. In addition to that, abscesses with negative cultures have been connected with higher mortality rates ${ }^{19}$, probably due to inadequate antimicrobial therapy.

The advances in neurosurgical techniques and the development of last generation antibiotics have contributed to decrease the morbidity and mortality associated with brain abcesses ${ }^{18,20}$. Furthermore, early diagnosis is related to better prognosis ${ }^{21}$. The adequate management of associated diseases, early treatment of infectious complications, and a high index of suspicion for the diagnosis will probably lead to a favorable outcome. This case report contributes to our better understanding of this condition, which might be amenable to cure by means of early detection and vigorous management, including surgical and clinical measures.

\section{REFERENCES}

1. Walker AE, Robins M, Weinfeld FD. Clinical findings. In: Weinfeld FD (Ed). National survey of stroke. Stroke 1981;12(Supl 1):S13-S31.

2. Beloosesky Y, Streifler JY, Eynan N, Grinblat J. Brain abscess complicating cerebral infarct. Age Ageing 2002;31:477-480.

3. Chen ST, Tang LM, Ro LS. Brain abscess as a complication of stroke. Stroke 1995;26:696-698.

4. Shu-Yuan Y. Brain abscess associated with congenital heart disease. Surg Neurol 1989;31:129-132.

5. Davenport RJ, Dennis MS, Wellwood I, Warlow CP. Complications after acute stroke. Stroke 1996:27:415-420.

6. Emejulu JK, Shokunbi MT, Malomo AO. Intracerebral abscesses: outcome following management in the CT era. West Afr J Med 2004;23:54-57.
7. Tunkel AR, Wispelwey B, Scheld WM. Brain abscess. In: Mandell GL, Bennett JE, Dolin R (Eds). Mandell, Gouglas, and Bennet's: principle and pratice of infectious diseases. $5^{\text {th }} \mathrm{Ed}$. Vol 1, Philadelphia: Churchill Livingstone, 2000:1016-1028.

8. Ni YH, Yeh KM, Peng MY, Chou YY, Chang FY. Community-acquired brain abscess in Taiwan: etiology and probable source of infection. J Microbiol Immunol Infect 2004;37:231-235.

9. Kaplan M, Ozveren F, Erol F, Kaplan S, Bilge T. Brain abscess developing at the site of preceding stroke. Report of three cases: review of literature. Neurosurgery 2005;15:17-20.

10. Amonn F, Muller U. Brain abscess: a possible complication of cerebral infarction? Schweiz Med Wochenschr 1984;114:58-62.

11. Ichimi K, Ishiguri H, Kida Y, Komomoto T. Brain abscess following cerebral infarction: a case report. No-Shinkei Geka 1989;17:381-385.

12. Inamasu J, Kagami H, Nakamura $Y$, et al. Brain abscess developing at site of preceding intracerebral hemorrhage. J Neurol 2002;249:221-223.

13. Lee KS, Bae WK, Bae HG, et al. Brain abscess from a ganglionic hemorrhage- a case report. J Korean Med Sci 1994;9:259-263.

14. Miyazaki H, Ito S, Nitta Y, Lino N, Shiokawa. Brain abscess following cerebral infarction. Acta Neurochir (Wien) 2004;146:531-532.

15. Okami N, Kawamata T, Sasahara A, et al. Brain abscess following thalamic hemorrhage: a case report. No Shinkei Geka 200;28:275-279.

16. Kerin MJ, Greenstein D, Chisholm EM et al. Is antibiotic penetration compromised in the ischemic tissues of patients undergoing amputation? Ann R Coll Surg Engl 1992;74:274-276.

17. Rayamarkers JT, Houben AJ, Van der Heyden JJ, et al. The effects of diabetes and severe ischemia on the penetration of ceftazidime into tissues of the limb. Diabet Med 2001;18:229-234.

18. Mampalam TJ, Rosenblum ML. Trends in the management of bacterial brain abscesses: a review of 102 cases over 17 years. Neurosurgery 1988;23:451-458.

19. Szuwart U, Bennefeld H. Bacteriological analysis of pyogenic infections of the brain. Neurosurg Rev 1990;13:113-118.

20. Yang SY, Zhao CS. Review of 140 patients with brain abscess. Surg Neurol. 1993;39:290-296.

21. Rappaport ZH, Vajda J. Intracranial abscess: current concepts in management. Neurosurgery 2002;12:238-250. 